\title{
ON THE CONCENTRATION OF CERTAIN ADDITIVE FUNCTIONS
}

\author{
DIMITRIS KOUKOULOPOULOS
}

In memoriam Jonas Kubilius

\begin{abstract}
We study the concentration of the distribution of an additive function, when the sequence of prime values of $f$ decays fast and has good spacing properties. In particular, we prove a conjecture by Erdös and Kátai on the concentration of $f(n)=\sum_{p \mid n}(\log p)^{-c}$ when $c>1$.
\end{abstract}

\section{INTRODUCTION}

An arithmetic function $f: \mathbb{N} \rightarrow \mathbb{R}$ is called additive if $f(m n)=f(m)+f(n)$ whenever $(m, n)=1$. According to the Kubilius probabilistic model of the integers, statistical properties of additive functions can be modeled by statistical properties of sums of independent random variables. We describe this model in the case that $f$ is a strongly additive function, that is to say $f$ satisfies the relation $f(n)=\sum_{p \mid n} f(p)$; the general case is slightly more involved. Let $\mathbb{P}$ denote the set of prime numbers and consider a sequence of independent Bernoulli random variables $\left\{X_{p}: p \in \mathbb{P}\right\}$ such that

$$
\operatorname{Prob}\left(X_{p}=1\right)=\frac{1}{p} \quad \text { and } \operatorname{Prob}\left(X_{p}=0\right)=1-\frac{1}{p} .
$$

The random variable $X_{p}$ can be thought as a model of the characteristic function of the event $\{n \in \mathbb{N}: p \mid n\}$. Then a probabilistic model for $f$ is given by the random variable $\sum_{p} f(p) X_{p}$.

The above model and well-known facts from probability theory lead to the prediction that the values of $f$ follow a certain distribution, possibly after rescaling them appropriately. In fact, the Erdős-Wintner theorem [8] states that if the series

$$
\sum_{|f(p)| \leq 1} \frac{f(p)}{p}, \quad \sum_{|f(p)| \leq 1} \frac{f^{2}(p)}{p}, \quad \sum_{|f(p)|>1} \frac{1}{p}
$$

converge, then $f$ has a limiting distribution, in the sense that there is a distribution function $F: \mathbb{R} \rightarrow[0,1]$ such that

$$
F_{x}(u):=\frac{1}{\lfloor x\rfloor}\{n \leq x: f(n) \leq u\} \mid \rightarrow F(u) \quad \text { as } \quad x \rightarrow \infty
$$

for every $u \in \mathbb{R}$ that is a point of continuity of $F$; the characteristic function of $F$ is given by

$$
\hat{F}(\xi)=\prod_{p}\left\{\left(1-\frac{1}{p}\right) \sum_{k \geq 0} \frac{e^{i \xi f\left(p^{k}\right)}}{p^{k}}\right\} .
$$

Conversely, if $f$ possesses a limiting distribution, then the three series in (1.1) converge.

Date: October 23, 2018.

2010 Mathematics Subject Classification. Primary: 11N60, 11 K65. 
One way to measure the regularity of the distribution of the set $\{f(n): n \in \mathbb{N}\}$ is by its concentration. In general, given a distribution function $G: \mathbb{R} \rightarrow[0,1]$, we define its concentration function to be

$$
Q_{G}(\epsilon)=\sup _{u \in \mathbb{R}}\{G(u+\epsilon)-G(u)\} .
$$

We seek estimates for $Q_{F_{x}}(\epsilon)$, or for $Q_{F}(\epsilon)$ if $f$ possesses a limiting distribution. There are various such results in the literature, a historic account of which is given in [1]. The most general estimate on $Q_{F_{x}}(\epsilon)$ is due to Ruzsa [12]. Improving upon bounds due to Erdös [5] and Halász [9], he showed that

$$
Q_{F_{x}}(1) \ll \max _{\lambda \in \mathbb{R}} \frac{1}{\sqrt{\lambda^{2}+\sum_{p \leq x} \min \left\{1,(f(p)-\lambda \log p)^{2}\right\} / p}} .
$$

This result is best possible, as can be seen by taking $f(n)=c \log n$ or $f(n)=\omega(n)=\sum_{p \mid n} 1$. However, both of these functions satisfy $f(p) \gg 1$. So, a natural question is whether it is possible to improve upon (1.2) in the case that $f(p)$ decays to zero. Erdős and Kátai [7], building on earlier work of Tjan [14] and Erdös [6], showed the following result:

Theorem 1.1 (Erdős, Kátai [7]). Let $f: \mathbb{N} \rightarrow \mathbb{R}$ be an additive function such that

$$
\sum_{p>t^{A}} \frac{|f(p)|}{p} \ll \frac{1}{t} \quad(t \geq 1), \quad\left|f\left(p_{1}\right)-f\left(p_{2}\right)\right| \gg \frac{1}{p_{2}^{B}} \quad\left(p_{1}, p_{2} \in \mathbb{P}, p_{1}<p_{2}\right),
$$

for some constants $A$ and $B$. Then

$$
Q_{F}(\epsilon) \asymp_{A, B} \frac{1}{\log (1 / \epsilon)} \quad(0<\epsilon \leq 1 / 2) ;
$$

except for $A$ and $B$, the implied constant depends on the implied constants in the assumptions of the theorem too.

On the other hand, when $f(p) \ll 1 / p^{\delta}, p \in \mathbb{P}$, for some $\delta>0$, then (1.2) applied to $f / \epsilon$ yields an upper bound for $Q_{F}(\epsilon)$ that is never better than $1 / \sqrt{\log \log (1 / \epsilon)}$, as can be seen by taking $\lambda=0$.

Also, Erdős and Kátai studied $Q_{F}(\epsilon)$ in the case that $f(p)=(\log p)^{-c}, p \in \mathbb{P}$, for some $c \geq 1$. They showed that

$$
\begin{cases}\epsilon^{1 / c} \ll_{c} Q_{F}(\epsilon) \ll_{c} \epsilon^{1 / c} \log \log ^{2}(1 / \epsilon) & \text { if } c>1 \\ \epsilon \ll Q_{F}(\epsilon) \ll \epsilon \log (1 / \epsilon) \log \log ^{2}(1 / \epsilon) & \text { if } c=1\end{cases}
$$

for $0<\epsilon \leq 1 / 3$. Furthermore, they conjectured that, for fixed every $c>1$, we have that

$$
Q_{F}(\epsilon) \asymp_{c} \epsilon^{1 / c} \quad(0<\epsilon \leq 1) .
$$

The conjecture of Erdős and Kátai was proven for $c$ large enough by La Bretèche and Tenenbaum in [1]:

Theorem 1.2 (La Bretèche, Tenenbaum [1]). Let $c \geq 1$ and $f: \mathbb{N} \rightarrow \mathbb{R}$ be an additive function such that $|f(p)| \asymp(\log p)^{-c}$ for every $p \in \mathbb{P}$ and

$$
\left|f\left(p_{1}\right)-f\left(p_{2}\right)\right| \gg \frac{p_{2}-p_{1}}{p_{2}\left(\log p_{2}\right)^{c+1}} \quad\left(p_{1}, p_{2} \in \mathbb{P}, p_{1}<p_{2}\right) .
$$


If $c$ is large enough, then we have that

$$
Q_{F}(\epsilon) \asymp \epsilon^{1 / c} \quad(0<\epsilon \leq 1) ;
$$

the implied constant depends at most on the implied constants in the assumptions of the theorem.

La Bretèche and Tenenbaum derived their theorem from a general upper bound on $Q_{F}(\epsilon)$ that they showed when the sequence of prime values of $f$ satisfies certain regularity assumptions. Their method uses a result from the theory of functions of Bounded Mean Oscillation, first introduced by Diamond and Rhoads 22 in this context to study the concentration of $f(n)=\log (\phi(n) / n)$.

In this paper we give a proof of the full Erdős-Kátai conjecture using a more elementary method, similar to the ones in [6, 7]:

Theorem 1.3. Let $c \geq 1$ and $f: \mathbb{N} \rightarrow \mathbb{R}$ be an additive function with $f(p)=(\log p)^{-c}$ for all $p \in \mathbb{P}$. For $0<\epsilon \leq 1 / 2$ we have that

$$
\epsilon^{1 / c} \ll Q_{F}(\epsilon) \ll \min \left\{\frac{c}{c-1}, \log \frac{1}{\epsilon}\right\} \epsilon^{1 / c} .
$$

Remark 1.4. When $0<c<1$, the behavior of $Q_{F}$ for $f$ as in Theorem 1.3 is different. As Gérald Tenenbaum has pointed out to us in a private communication, in this case we have that

$$
Q_{F}(\epsilon) \asymp_{c} \epsilon \quad(0<\epsilon \leq 1) .
$$

Corollary 1.3 and relation (1.4) give the concentration of an additive function $f$ with $f(p)=$ $(\log p)^{-c}, p \in \mathbb{P}$, for all positive values of $c$ except for $c=1$, which is the only case remaining open.

We will prove Theorem 1.3 in Section 2, The method of its proof is quite flexible; in particular, it leads to a strengthening of Theorems 1.1 and 1.2. We phrase our more general result in terms of the distribution function

$$
\mathcal{F}_{y}(u)=\prod_{p \leq y}\left(1-\frac{1}{p}\right) \sum_{\substack{p \mid n \Rightarrow p \leq y \\ f(n) \leq u}} \frac{1}{n} \quad(u \in \mathbb{R}),
$$

defined for every $y \geq 1$. From a technical point of view, this function is more natural to work with than $F_{x}$. Indeed, a calculation of the characteristic function of $\mathcal{F}_{y}$ immediately implies that $\mathcal{F}_{y}$ converges to $F$ weakly, provided that the latter is well defined. It is relatively easy to pass from estimates for $Q_{\mathcal{F}_{y}}(\epsilon)$ to estimates for $Q_{F_{x}}(\epsilon)$.

With this notation, we have the following result (observe that by letting $y \rightarrow \infty$ in it, we deduce as special cases 1 Theorems 1.1 and 1.2):

Theorem 1.5. Consider an additive function $f: \mathbb{N} \rightarrow \mathbb{R}$ for which there is a set of primes $\mathcal{P}$ and a constant $c \in[1,2]$ such that

$$
|f(p)| \ll \frac{1}{(\log p)^{c}} \quad(p \in \mathcal{P}), \quad \text { and } \quad \sum_{p \in \mathbb{P} \backslash \mathcal{P}} \frac{1}{p} \ll 1 .
$$

\footnotetext{
${ }^{1}$ To deduce Theorem 1.1, take $\mathcal{P}=\left\{p \in \mathbb{P}:|f(p)| \leq p^{-1 /(2 A)}\right\}$.
} 
For $t \geq 2$ set

$$
g(t)=\frac{\sup \left\{|f(p)|(\log p)^{c}: p \geq t, p \in \mathcal{P}\right\}}{(\log t)^{c}}
$$

and assume that there is some $A \geq 1$ such that

$$
\left|f\left(p_{2}\right)-f\left(p_{1}\right)\right| \gg \min \left\{\frac{g\left(p_{2}\right)\left(p_{2}-p_{1}\right)}{p_{2} \log p_{2}}, g\left(p_{2}^{A}\right)\right\} \quad\left(p_{1}, p_{2} \in \mathcal{P}, p_{1}<p_{2}\right) .
$$

Then for $0<\epsilon \leq 1 / 2$ and $y \geq K(\epsilon)$, where $K(\epsilon)=\min \{p \in \mathbb{P}: g(p) \leq \epsilon\}$, we have that

$$
\frac{1}{\log K(\epsilon)} \ll Q_{\mathcal{F}_{y}}(\epsilon) \ll_{A} \frac{\min \{1 /(c-1), \log (1 / \epsilon)\}}{\log K(\epsilon)} ;
$$

except for $A$, the implied constants depends on the implied constants in the assumptions of the theorem too.

As an immediate corollary, we deduce the following simpler to state result.

Corollary 1.6. Let $f: \mathbb{N} \rightarrow \mathbb{R}$ be an additive function for which there is a constant $c \in[1,2]$ such that the sequence $\left\{|f(p)|(\log p)^{c}: p\right.$ prime $\}$ is decreasing. Then for $0<\epsilon \leq 1 / 2$ and $y \geq K(\epsilon)$, where $K(\epsilon)=\min \{p \in \mathbb{P}:|f(p)| \leq \epsilon\}$, we have that

$$
\frac{1}{\log K(\epsilon)} \ll Q_{\mathcal{F}_{y}}(\epsilon) \ll \frac{\min \{1 /(c-1), \log (1 / \epsilon)\}}{\log K(\epsilon)} .
$$

Proof. If $g$ is as in the statement of Theorem 1.5 with $\mathcal{P}=\mathbb{P}$, then we see immediately that $g(p)=|f(p)|$, for all $p \in \mathbb{P}$. Moreover, if $p_{1}<p_{2}$ are two primes, then we have that

$$
\begin{aligned}
\frac{\left|f\left(p_{2}\right)-f\left(p_{1}\right)\right|}{\left|f\left(p_{2}\right)\right|} \geq \frac{\left|f\left(p_{1}\right)\right|}{\left|f\left(p_{2}\right)\right|}-1 \geq \frac{\left(\log p_{2}\right)^{c}}{\left(\log p_{1}\right)^{c}}-1 & =\frac{\left(\log p_{2}\right)^{c}-\left(\log p_{1}\right)^{c}}{\left(\log p_{1}\right)^{c}} \\
& \geq \frac{c\left(p_{2}-p_{1}\right)\left(\log p_{1}\right)^{c-1}}{p_{2}\left(\log p_{1}\right)^{c}} \\
& \geq \frac{p_{2}-p_{1}}{p_{2} \log p_{2}},
\end{aligned}
$$

by our assumption that $\left\{|f(p)|(\log p)^{c}: p\right.$ prime $\}$ is decreasing and the Mean Value Theorem. So Theorem 1.5 can be applied and the claimed result follows.

The lower bound in Theorem 1.5, which will be proven in Section 3, is a straightforward application of Theorem 1.2 in [1]. On the other hand, for the proof of the upper bound in Theorem 1.5, which will be given in Section 4, we use a combination of ideas from [6, 7]. Even though Theorem 1.3 is an immediate corollary of Theorem 1.5, applied with min $\{c, 2\}$ in place of $c$, we have chosen to give the proof of both of them in full detail, so that to motivate certain choices in the proof of Theorem 1.5, which is rather technical.

A heuristic argument. There is a simple heuristic argument which motivates Theorem 1.5. We demonstrate it in the simpler setting of Corollary 1.6, that is to say when the sequence $\left\{|f(p)|(\log p)^{c}: p \in \mathbb{P}\right\}$ is decreasing. For every integer $n$, we have that

$$
\sum_{p \mid n, p \geq K(\epsilon)}|f(p)| \leq \epsilon \sum_{p \mid n, p \geq K(\epsilon)} \frac{(\log K(\epsilon))^{c}}{(\log p)^{c}} .
$$


Since for a typical integer $n$ the sequence $\{\log \log p: p \mid n\}$ is distributed like an arithmetic progression of step 1 (see, for example, [10, Chapter 1]), we find that 2

$$
\sum_{p \mid n, p \geq K(\epsilon)}|f(p)| \lesssim \epsilon \sum_{j \geq \log \log K(\epsilon)} \frac{(\log K(\epsilon))^{c}}{e^{c j}} \ll \epsilon .
$$

So only the prime divisors of $n$ lying in $[1, K(\epsilon))$ are important for the size of $Q_{F}(\epsilon)$. Note that for a prime number $p<K(\epsilon)$, we have that $|f(p)|>\epsilon$. Therefore if $a$ and $b$ are composed of primes within $[1, K(\epsilon))$, then it is reasonable to expect that $|f(a)-f(b)|$ is big compared to $\epsilon$, unless $a$ and $b$ have a large common factor. This leads to the prediction that $Q_{F}(\epsilon) \approx 1 / \log K(\epsilon)$, which is confirmed by Theorem 1.5 when $c>1$. However, when $c<1$ this heuristic fails, as (1.4) shows, and the underlying reason is combinatorial: the pigeonhole principle implies the lower bound $Q_{F}(\epsilon) \gg_{F} \epsilon$ for the concentration function of any distribution function $F$ (see also [7, Remark 1, p. 297]).

Notation. For an integer $n$ we denote with $P^{+}(n)$ and $P^{-}(n)$ its largest and smallest prime factors, respectively, with the notational convention that $P^{+}(1)=1$ and $P^{-}(1)=\infty$. The symbols $p$ and $p^{\prime}$ always denote prime numbers. The set of all primes numbers is denoted by $\mathbb{P}$. Finally, given $\mathcal{P} \subset \mathbb{P}$ and real numbers $1 \leq z \leq w$, we write $\mathcal{P}(z, w)$ for the set of integers all of whose prime factors belong to $\mathcal{P} \cap(z, w]$.

\section{The CONJECTURE OF ERDŐS AND KÁtAI}

Proof of Theorem 1.3. The lower bound follows by relation (1.3), with the implied constant depending on $c$. To remove this dependence, see Theorem 3.1 below.

It remains to show the corresponding upper bound. Before delving into the details of the proof, we give a brief outline of the main idea. For $\delta>0$, we set $P_{\delta}=\exp \left\{\delta^{-1 / c}\right\}$, so that $f(p)=\delta$ if and only if $p=P_{\delta}$. As the heuristic argument presented towards the end of Section 1 indicates, it suffices to bound $Q_{\mathcal{F}_{q}}(\epsilon)$, where $q:=P_{2 \epsilon}$. We split the elements of the set $\mathcal{M}:=\{n \in \mathbb{P}(1, q): u<f(n) \leq u+\epsilon\}$ into subsets $\mathcal{M}_{\delta}:=\left\{n \in \mathcal{M}: P_{2 \delta}<P^{-}(n) \leq P_{\delta}\right\}$, where $\delta \in\left\{2^{j} \epsilon: 1 \leq j \leq j_{0}\right\}$ with $\left.j_{0}=\lfloor(\log (1 / \epsilon)-c \log \log 2) / \log 2\rfloor\right\}$. Then we find that

$$
\sum_{n \in \mathcal{M}_{\delta}} \frac{1}{n} \approx \sum_{m \in \mathbb{P}\left(P_{\delta}, q\right)} \frac{1}{m} \sum_{\substack{P_{2 \delta}<p \leq P_{\delta} \\ u-f(m)<f(p) \leq u-f(m)+\epsilon}} \frac{1}{p} .
$$

Fix $m$ for the moment and set $v_{m}=u-f(m)$. Then the variable $p$ lies in the interval $I_{m}=\left[P_{\min \left\{v_{m}+\epsilon, 2 \delta\right\}}, P_{\max \left\{v_{m}, \delta\right\}}\right]$, which is non-empty only when $v_{m}+\epsilon \geq \delta \geq v_{m} / 2$. Since $\delta \geq 2 \epsilon$ by assumption, we find that $v_{m} \asymp \delta \gg \epsilon$. So the interval $I_{m}$ has double-logarithmic length $3^{3} \ll \epsilon / v_{m} \asymp \epsilon / \delta$. Hence the Prime Number Theorem [13, Theorem 1, p. 167] implies that $\sum_{p \in I_{m}} 1 / p \lesssim \epsilon / \delta$, provided that $I$ is not too short. Assuming that this is indeed the case, we deduce that

$$
\sum_{n \in \mathcal{M}_{\delta}} \frac{1}{n} \lesssim \frac{\epsilon}{\delta} \sum_{m \in \mathbb{P}\left(P_{\delta}, q\right)} \frac{1}{m} \ll \frac{\epsilon \log q}{\delta \log P_{\delta}} \asymp \frac{\epsilon^{1-1 / c}}{\delta^{1-1 / c}} .
$$

\footnotetext{
${ }^{2}$ The symbol ' $\lesssim$ ' here is used in a non-rigorous fashion to denote 'roughly less than'. Similarly, the symbol ' $\approx$ ' means 'roughly equal to'.

${ }^{3}$ Given an interval $I=[\alpha, \beta]$, its double-logarithmic length is $\log \log \beta-\log \log \alpha$.
} 
Summing the above inequality over $\delta \in\left\{2^{j} \epsilon: 1 \leq j \leq j_{0}\right\}$ implies that

$$
\begin{aligned}
Q_{F}(\epsilon) \approx Q_{\mathcal{F}_{q}}(\epsilon) \lesssim \frac{1}{\log q} \sum_{j=1}^{j_{0}} \sum_{n \in \mathcal{M}_{2 j}} \frac{1}{n} & \lesssim \epsilon^{1 / c} \sum_{j=1}^{j_{0}} \frac{1}{2^{j(1-1 / c)}} \\
& \ll \min \left\{\frac{c}{c-1}, \log \frac{1}{\epsilon}\right\} \epsilon^{1 / c},
\end{aligned}
$$

which shows (heuristically at least) the desired result.

The main technical difficulty we have to surpass in order to make the above argument work is that the estimate $\sum_{p \in I_{m}} 1 / p \ll \epsilon / \delta$, which we used above, might not be accurate for large $\delta$ (i.e. when $P_{\delta}$ is small). So below we shall employ a variation of the argument of this paragraph where, instead of looking where $P^{-}(n)$ lies, we will look where $\min \left\{p \mid n: p \geq q^{\prime}\right\}$ lies, with $q^{\prime}$ being some small parameter chosen appropriately.

Without loss of generality, we may assume that $\epsilon \leq 1 / 100^{c}$; otherwise (2.2) follows immediately by the trivial bound $Q_{F}(\epsilon) \leq 1$. Define $\eta$ by $P_{\eta} / \eta^{2}=1 / \epsilon^{2}$. Note that $4 \epsilon \leq \eta \leq 1 / 2$, since $P_{4 \epsilon} /(4 \epsilon)^{2} \geq 1 / \epsilon^{2} \geq P_{1 / 2} /(1 / 2)^{2}$ by our assumption that $\epsilon \leq 1 / 100^{c}$. Before we proceed further, we will prove that, for $v \in \mathbb{R}, \delta \in[2 \epsilon, 1]$ and $2 \leq z \leq P_{\delta}$, we have that

$$
\sum_{\substack{z<p \leq P_{\delta} \\ v<f(p) \leq v+\epsilon}} \frac{1}{p} \ll \frac{\epsilon}{\delta}+\frac{1}{\sqrt{z}} \ll \begin{cases}\epsilon / \delta & \text { if } z \geq P_{2 \delta} \geq P_{\eta} \\ \epsilon / \delta+1 /(\log z)^{2} & \text { otherwise. }\end{cases}
$$

First, note that it suffices to show the first inequality. Indeed, if $z \geq P_{2 \delta} \geq P_{\eta}$, then $P_{2 \delta} /(2 \delta)^{2} \geq P_{\eta} / \eta^{2}=1 / \epsilon^{2}$ and thus $\sqrt{z} \geq \sqrt{P_{2 \delta}} \geq 2 \delta / \epsilon$, which proves the second inequality of (2.1). Turning back to the first inequality of (2.1), observe that the primes $p$ on the left hand side of (2.1) lie in the interval $\left[\max \left\{z, P_{v+\epsilon}\right\}, P_{\max \{v, \delta\}}\right]=:[\alpha, \beta]$. For this interval to be non-empty we need that $v+\epsilon \geq \delta$. Since $\delta \geq 2 \epsilon$, we deduce that $v \geq \epsilon$ and thus $2 v \geq v+\epsilon \geq \delta$. So we have that

$$
\log \left(\frac{\log \beta}{\log \alpha}\right) \leq \log \left(\frac{\log P_{v}}{\log P_{v+\epsilon}}\right)=\frac{1}{c} \log \left(\frac{v+\epsilon}{v}\right) \leq \frac{\epsilon}{v} \leq \frac{2 \epsilon}{\delta} .
$$

Thus, if $\beta \geq \alpha+\sqrt{\alpha}$, then covering the interval $[\alpha, \beta]$ by subintervals of the form $[y, y+\sqrt{y})$ and applying the Brun-Titchmarsch inequality [13, Theorem 9, p. 73] to each one of them yields that

$$
\sum_{\substack{z<p \leq P_{\delta} \\ v<f(p) \leq v+\epsilon}} \frac{1}{p} \ll \frac{\epsilon}{\delta}
$$

which proves (2.1). Finally, if $\beta<\alpha+\sqrt{\alpha}$, then we have that

$$
\sum_{\substack{z<p \leq P_{\delta} \\ v<f(p) \leq v+\epsilon}} \frac{1}{p} \leq \sum_{\alpha \leq p \leq \beta} \frac{1}{p} \leq \frac{\beta-\alpha+2}{\alpha} \ll \frac{1}{\sqrt{\alpha}} \leq \frac{1}{\sqrt{z}}
$$

and (2.1) follows in this last case too.

We are now ready to show the upper bound implicit in Theorem 1.3. Fix for the moment $y \geq q=P_{2 \epsilon}$ and $u \in \mathbb{R}$. Given $n \in \mathbb{P}(1, y)$ with $u<f(n) \leq u+\epsilon$, we write $n=a b$, 
where $a$ is square-free, $b$ is square-full and $(a, b)=1$. We further decompose $a=a_{1} a_{2}$, where $P^{+}\left(a_{1}\right) \leq q<P^{-}\left(a_{2}\right)$. So

$$
\begin{aligned}
\sum_{\substack{P^{+}(n) \leq y \\
u<f(n) \leq u+\epsilon}} \frac{1}{n} & =\sum_{\substack{P^{+}(b) \leq y \\
b \text { square-full }}} \frac{1}{b} \sum_{\substack{a_{2} \in \mathbb{P}(q, y) \\
\left(a_{2}, b\right)=1}} \frac{\mu^{2}\left(a_{2}\right)}{a_{2}} \sum_{\substack{P^{+}\left(a_{1}\right) \leq q,\left(a_{1}, b\right)=1 \\
u-f\left(a_{2} b\right)<f\left(a_{1}\right) \leq u-f\left(a_{2} b\right)+\epsilon}} \frac{\mu^{2}\left(a_{1}\right)}{a_{1}} \\
& \leq \sum_{\substack{P^{+}(b) \leq y \\
b \text { square-full }}} \frac{1}{b} \sum_{a_{2} \in \mathbb{P}(q, y)} \frac{1}{a_{2}} \sup _{v \in \mathbb{R}}\left\{\sum_{\substack{P^{+}\left(a_{1}\right) \leq q \\
v<f\left(a_{1}\right) \leq v+\epsilon}} \frac{\mu^{2}\left(a_{1}\right)}{a_{1}}\right\} \\
& \ll \frac{\log y}{\log q} \cdot \sup _{v \in \mathbb{R}}\left\{\sum_{\substack{P^{+}\left(a_{1}\right) \leq q \\
v<f\left(a_{1}\right) \leq v+\epsilon}} \frac{\mu^{2}\left(a_{1}\right)}{a_{1}}\right\} .
\end{aligned}
$$

Since $\log q \asymp \epsilon^{-1 / c}$, then Theorem 1.3 will follow from the estimate

$$
\sum_{\substack{P^{+}(n) \leq q \\ v<f(n) \leq v+\epsilon}} \frac{\mu^{2}(n)}{n} \ll \min \left\{\frac{c}{c-1}, \log \frac{1}{\epsilon}\right\} \quad(v \in \mathbb{R})
$$

by letting $y \rightarrow \infty$. Set $J=-1+\lfloor\log (\eta / \epsilon) / \log 2\rfloor \in \mathbb{N}$ and, for $j \geq 0$, define $q_{j}=P_{2^{j+1} \epsilon}$, so that $q=q_{0}>\cdots>q_{J} \geq P_{\eta}$. Fix $v \in \mathbb{R}$ and let

$$
\mathcal{N}=\left\{n \in \mathbb{N}: \mu^{2}(n)=1, P^{+}(n) \leq q_{0}, v<f(n) \leq v+\epsilon\right\}
$$

As in the heuristic argument of the first paragraph, we partition $\mathcal{N}$ into certain subsets and estimate the contribution of each one of them to $\sum_{n \in \mathcal{N}} 1 / n$ separately. The difference is that instead of looking at the location of $P^{-}(n)$, we write $n=a n^{\prime}$ with $P^{+}(a) \leq q_{J}<P^{-}\left(n^{\prime}\right)$ and look at the location of $p=P^{-}\left(n^{\prime}\right)$. An additional fact that we shall take advantage of is that if $n^{\prime}=p b$ and $\log p \asymp \log P_{\delta}$, then, for fixed $b$, the number $f(a)$ lies in an interval of length $\ll \epsilon+f(p) \ll \epsilon+\delta \ll \delta$, which allows us to gain an additional crucial savings in our estimate for $\sum_{n \in \mathcal{N}} 1 / n$. So we write $\mathcal{N}=\cup_{j=0}^{J} \mathcal{N}_{j}$, where $\mathcal{N}_{0}=\left\{n \in \mathcal{N}: P^{+}(n) \leq q_{J}\right\}$ and

$$
\mathcal{N}_{j}=\left\{n \in \mathcal{N}: n=a p b, P^{+}(a) \leq q_{J}<p<P^{-}(b), q_{j}<p \leq q_{j-1}\right\}
$$

for $j \in\{1, \ldots, J\}$.

First, we bound $\sum_{n \in \mathcal{N}_{0}} 1 / n$. If $n>1$, then we write $n=m P^{+}(n)=m p^{\prime}$. Thus

$$
\begin{aligned}
\sum_{n \in \mathcal{N}_{0}} \frac{1}{n} & \leq 1+\sum_{P^{+}(m) \leq q_{J}} \frac{1}{m} \sum_{\substack{P^{+}(m)<p^{\prime} \leq q_{J} \\
v-f(m)<f\left(p^{\prime}\right) \leq v-f(m)+\epsilon}} \frac{1}{p^{\prime}} \\
& \ll 1+\sum_{P^{+}(m) \leq q_{J}} \frac{1}{m}\left(\frac{\epsilon}{2^{J} \epsilon}+\frac{1}{\log ^{2}\left(1+P^{+}(m)\right)}\right) \ll 1+\frac{\log q_{J}}{2^{J}},
\end{aligned}
$$

by relation (2.1). 
Next, we bound $\sum_{n \in \mathcal{N}_{j}} 1 / n$ for $j \in\{1, \ldots, J\}$. We have that

$$
\begin{aligned}
\sum_{n \in \mathcal{N}_{j}} \frac{1}{n} & \leq \sum_{b \in \mathbb{P}\left(q_{j}, q_{0}\right)} \frac{1}{b} \sum_{P^{+}(a) \leq q_{J}} \frac{\mu^{2}(a)}{a} \sum_{\substack{q_{j}<p \leq q_{j-1} \\
v-f(a)-f(b)<f(p) \leq v-f(a)-f(b)+\epsilon}} \frac{1}{p} \\
& \leq \sum_{b \in \mathbb{P}\left(q_{j}, q_{0}\right)} \frac{1}{b} \sup _{w \in \mathbb{R}}\left\{\sum_{P^{+}(a) \leq q_{J}} \frac{\mu^{2}(a)}{a} \sum_{\substack{q_{j}<p \leq q_{j-1} \\
w-f(a)<f(p) \leq w-f(a)+\epsilon}} \frac{1}{p}\right\} \\
& \ll \frac{\log q_{0}}{\log q_{j}} \cdot \sup _{w \in \mathbb{R}}\left\{\sum_{P^{+}(a) \leq q_{J}} \frac{\mu^{2}(a)}{a} \sum_{\substack{q_{j}<p \leq q_{j-1} \\
w-f(a)<f(p) \leq w-f(a)+\epsilon}} \frac{1}{p}\right.
\end{aligned}
$$

Fix some $w \in \mathbb{R}$ and consider $a$ with $P^{+}(a) \leq q_{J}$ and $p \in\left(q_{j}, q_{j-1}\right]$ with $w<f(a)+f(p) \leq$ $w+\epsilon$, as above. Since $|f(p)| \leq 2^{j+1} \epsilon$ for $p>q_{j}$, we must have that $|f(a)-w|<2^{j+2} \epsilon$. So

$$
\begin{aligned}
\sum_{P^{+}(a) \leq q_{J}} \frac{\mu^{2}(a)}{a} \sum_{\substack{q_{j}<p \leq q_{j-1} \\
w-f(a)<f(p) \leq w-f(a)+\epsilon}} \frac{1}{p}=\sum_{\substack{P^{+}(a) \leq q_{J} \\
|f(a)-w|<2^{j+2} \epsilon}} \frac{\mu^{2}(a)}{a} \sum_{\substack{q_{j}<p \leq q_{j-1} \\
w-f(a)<f(p) \leq w-f(a)+\epsilon}} \frac{1}{p} \\
\ll \frac{\epsilon}{2^{j} \epsilon} \sum_{\substack{P^{+}(a) \leq q_{J} \\
|f(a)-w|<2^{j+2} \epsilon}} \frac{\mu^{2}(a)}{a},
\end{aligned}
$$

by the first part of (2.1) applied with $w-f(a), 2^{j} \epsilon$ and $q_{j}$ in place of $v, \delta$ and $z$, respectively, since $q_{j} \geq q_{J} \geq P_{\eta}$. Finally, if $a>1$, then we write $a=m P^{+}(a)=m p^{\prime}$. So we find that

$$
\sum_{\substack{P^{+}(a) \leq q_{J} \\|f(a)-w|<2^{j+2} \epsilon}} \frac{\mu^{2}(a)}{a} \leq 1+\sum_{P^{+}(m) \leq q_{J}} \frac{1}{m} \sum_{\substack{P^{+}(m)<p^{\prime} \leq q_{J} \\\left|f\left(p^{\prime}\right)-(w-f(m))\right|<2^{j+2} \epsilon}} \frac{1}{p^{\prime}} .
$$

For every fixed $m \in \mathbb{N}$, we have that

$$
\sum_{\substack{P^{+}(m)<p^{\prime}<q_{J} \\\left(p^{\prime}\right)-(w-f(m)) \mid<2^{j+2} \epsilon}} \frac{1}{p^{\prime}} \ll \frac{2^{j} \epsilon}{2^{J} \epsilon}+\frac{1}{\log ^{2}\left(1+P^{+}(m)\right)},
$$

by the second part of (2.1) with $2^{j} \epsilon, 2^{J+1} \epsilon$ and $P^{+}(m)$ in place of $\epsilon, \delta$ and $z$, respectively, and with $v \in\left\{w-f(m)+h \cdot 2^{j} \epsilon: h \in[-4,4) \cap \mathbb{Z}\right\}$. So we find that

$$
\sum_{\substack{P^{+}(a) \leq q_{J} \\|f(a)-w|<2^{j+1} \epsilon}} \frac{\mu^{2}(a)}{a} \ll 1+\sum_{P^{+}(m) \leq q_{J}} \frac{1}{m}\left(\frac{2^{j} \epsilon}{2^{J} \epsilon}+\frac{1}{\log ^{2}\left(1+P^{+}(m)\right)}\right) \ll 1+\frac{\log q_{J}}{2^{J-j}} .
$$

\footnotetext{
${ }^{4}$ Note that the parameter $\eta$ is not involved in the second part, so the same proof allows us to replace $\epsilon$ with $2^{j} \epsilon$.
} 
Combining the above inequality with (2.5) and (2.6) $)$, we deduce that

$$
\sum_{n \in \mathcal{N}_{j}} \frac{1}{n} \ll \frac{1}{2^{j}}\left(1+\frac{\log q_{J}}{2^{J-j}}\right) \frac{\log q_{0}}{\log q_{j}} \ll \frac{1}{2^{j(1-1 / c)}}\left(1+\frac{\log q_{J}}{2^{J-j}}\right) .
$$

Together with relation (2.4), this implies that

$$
\begin{aligned}
\sum_{n \in \mathcal{N}} \frac{1}{n} \ll \sum_{j=0}^{J} \frac{1}{2^{j(1-1 / c)}}\left(1+\frac{\log q_{J}}{2^{J-j}}\right) & =\sum_{j=0}^{J} \frac{1}{2^{j(1-1 / c)}}+\sum_{j=0}^{J} \frac{2^{j / c} \log q_{J}}{2^{J}} \\
& \ll \min \left\{\frac{c}{c-1}, J\right\}+\frac{\min \{c, J\} \log q_{J}}{2^{J(1-1 / c)}}
\end{aligned}
$$

Furthermore, we have that $2^{J} \asymp \eta / \epsilon$ and, as a result,

$$
\log q_{J}=\left(2^{J+1} \epsilon\right)^{-1 / c} \asymp \eta^{-1 / c}=\log P_{\eta}=2 \log (\eta / \epsilon) \ll J \ll \log (1 / \epsilon) .
$$

Thus

$$
\begin{aligned}
\sum_{n \in \mathcal{N}} \frac{1}{n} \ll \min \left\{\frac{c}{c-1}, J\right\}+\frac{J \min \{c, J\}}{2^{J(1-1 / c)}} & \ll \min \left\{\frac{c}{c-1}, J\right\} \\
& \ll \min \left\{\frac{c}{c-1}, \log \frac{1}{\epsilon}\right\},
\end{aligned}
$$

by the inequality $2^{J(1-1 / c)} \gg J^{2}$ if $c \geq 2$ and the inequality $2^{J(1-1 / c)} \gg \max \{1, J(1-1 / c)\}$ if $1 \leq c \leq 2$. Therefore relation (2.2) follows, thus completing the proof of the theorem.

\section{The LOWER BOUND IN THEOREM 1.5}

In this section we derive the lower bound in Theorem 1.5 from the following general result, which is a corollary of Theorem 1.2 in [1].

Theorem 3.1. Let $f: \mathbb{N} \rightarrow \mathbb{R}$ be an additive function and $0<\epsilon<1$. If there is a set of primes $\mathcal{P}$ and some $M \geq 2$ such that

$$
\sum_{p \in \mathbb{P} \backslash \mathcal{P}} \frac{1}{p} \ll 1 \quad \text { and } \quad \sum_{p \in \mathcal{P}, p>M} \frac{|f(p)|}{p} \ll \epsilon,
$$

then, for $y \geq M$, we have that

$$
Q_{\mathcal{F}_{y}}(\epsilon) \gg \frac{1}{\log M}
$$

the implied constant depends at most on the implied constants implicit in the assumptions of the theorem.

Proof. Let $M_{0}$ be a large constant to be chosen later. If $M \leq y \leq M_{0}$, then the theorem follows by the trivial bound $Q_{\mathcal{F}_{y}}(\epsilon) \gg 1 / \log y$, which holds since 1 is always in $\{n \in \mathbb{N}$ : $\left.P^{+}(n) \leq y,|f(n)|<\epsilon / 2\right\}$. Assume now that $y \geq M_{0}$ and set $M^{\prime}=\max \left\{M, M_{0}\right\}$, so that $y \geq M^{\prime}$. Let

$$
C=\frac{1}{\epsilon} \sum_{p \in \mathcal{P}, p>M^{\prime}} \frac{|f(p)|}{p} \ll 1
$$


Define $g: \mathbb{N} \rightarrow \mathbb{R}$ by

$$
g(n)= \begin{cases}f(n) & \text { if } n \in \mathcal{P}(1, y), \\ 0 & \text { otherwise }\end{cases}
$$

and call $G$ its distribution function. Then Theorem 1.2 in [1] yields that 5

$$
Q_{G}(3 C \epsilon) \geq\left(1-2 \cdot \frac{C \epsilon}{3 C \epsilon}+o_{M^{\prime} \rightarrow \infty}(1)\right) \prod_{p \leq M^{\prime}}\left(1-\frac{1}{p}\right) \gg \frac{1}{\log M^{\prime}} .
$$

So, by the pigeonhole principle, we deduce that

$$
Q_{G}(\epsilon) \geq \frac{Q_{G}(3 C \epsilon)}{3 C+1} \gg_{C} \frac{1}{\log M^{\prime}} \asymp \frac{1}{\log M},
$$

provided that $M_{0}$ is large enough. Finally, we have that

$$
\begin{aligned}
Q_{G}(\epsilon) & =\sup _{u \in \mathbb{R}}\left\{\prod_{p \in \mathcal{P} \cap[1, y]}\left(1-\frac{1}{p}\right) \sum_{\substack{n \in \mathcal{P}(1, y) \\
u<f(n) \leq u+\epsilon}} \frac{1}{n}\right\} \\
& \leq Q_{\mathcal{F}_{y}}(\epsilon) \prod_{p \in \mathbb{P} \backslash \mathcal{P}}\left(1-\frac{1}{p}\right)^{-1} \ll Q_{\mathcal{F}_{y}}(\epsilon),
\end{aligned}
$$

which together with (3.1) completes the proof of the theorem.

Proof of the lower bound in Theorem 1.5. The definition of $g$ implies that the function $t \rightarrow$ $g(t)(\log t)^{c}$ is decreasing. Thus, for $p \in \mathcal{P}$ with $p \geq K(\epsilon)$, we have that

$$
|f(p)| \leq g(p) \leq \frac{g(K(\epsilon))(\log (K(\epsilon)))^{c}}{(\log p)^{c}} \leq \frac{\epsilon(\log (K(\epsilon)))^{c}}{(\log p)^{c}} .
$$

Consequently,

$$
\sum_{p>K(\epsilon)} \frac{|f(p)|}{p} \ll \epsilon
$$

which implies that the hypotheses of Theorem 3.1 are satisfied with $M=K(\epsilon)$ and $\mathcal{P}$, and the desired lower bound follows.

\section{The Upper Bound in Theorem 1.5}

We conclude the paper by showing the upper bound in Theorem 1.5. We start with the following technical lemma whose hypotheses mimic all the crucial facts about the additive function $f(n)=\sum_{p \mid n}(\log p)^{-c}$ that we used in the proof of Theorem 1.3.

Lemma 4.1. Let $f: \mathbb{N} \rightarrow \mathbb{R}$ be an additive function for which there is a set of primes $\mathcal{P}$ and a decreasing function $P_{f}:(0,1] \rightarrow[2,+\infty)$ such that

$$
\begin{gathered}
\sum_{p \in \mathbb{P} \backslash \mathcal{P}} \frac{1}{p} \ll 1, \\
|f(p)| \leq \epsilon \quad\left(0<\epsilon \leq 1, p \in \mathcal{P}, p>P_{f}(\epsilon)\right) .
\end{gathered}
$$

\footnotetext{
${ }^{5}$ In [1, Theorem 1.2], the authors let $\epsilon \rightarrow 0$. However, an easy modification of their proof allows us to let instead $M^{\prime} \rightarrow \infty$.
} 
Furthermore, assume that there is some $\lambda \in(0,1]$ and some $\rho \geq 1$ such that

$$
\sum_{\substack{p \in \mathcal{P} \cap(z, w] \\ u<f(p) \leq u+\epsilon}} \frac{1}{p} \ll \begin{cases}\epsilon / \delta & \text { if } z \geq P_{f}(2 \delta) \geq(2 \delta / \epsilon)^{\rho}, \\ \epsilon / \delta+1 /(\log z)^{2} & \text { otherwise, }\end{cases}
$$

for all $u \in \mathbb{R}, 0<\epsilon \leq \delta \leq 1$ and $2 \leq z \leq w \leq \min \left\{P_{f}(\delta), P_{f}(\epsilon)^{\lambda}\right\}$. Let $0<\epsilon \leq \delta \leq 1$ such that $P_{f}(\delta) \leq P_{f}(\epsilon)^{\lambda}$, set $q_{j}=P_{f}\left(2^{j} \delta\right)$ for $j \geq 0$, and consider $J \in\{0\} \cup\left\{j \in \mathbb{N}: 2^{j} \leq\right.$ $1 / \delta$ and $\left.q_{j} \geq\left(2^{j} \delta / \epsilon\right)^{\rho}\right\}$. For $y \geq q_{0}$, we have that

$$
Q_{\mathcal{F}_{y}}(\epsilon) \ll \frac{1}{\log q_{0}}+\frac{\epsilon}{\lambda \delta} \sum_{j=1}^{J} \frac{1}{2^{j} \log q_{j}}+\frac{\epsilon}{\delta} \sum_{j=0}^{J} \frac{\log q_{J}}{2^{J} \log q_{j}} ;
$$

the implied constant depends at most on the implied constants in (4.1) and (4.3).

Remark 4.2. The parameters $\lambda$ and $\rho$, and the set $\mathcal{P}$ are introduced to make Lemma 4.1 more applicable. One can think of $P_{f}$ defined by $P_{f}(\epsilon)=\max \{p \in \mathbb{P}:|f(p)|>\epsilon\}$. Condition (4.3) can be motivated as follows. Assume that

$$
\sum_{p>P_{f}(\alpha)} \frac{|f(p)|}{p} \ll \alpha \quad(0<\alpha \leq 1) .
$$

We have that $|f(p)| \approx \delta$ for $p \in\left(P_{f}(2 \delta), P_{f}(\delta)\right]$. So if the sequence $\{f(p): p \in \mathbb{P}\}$ is 'well-spaced', then we expect that

$$
\sum_{\substack{P_{f}(2 \delta)<p \leq P_{f}(\delta) \\ w<f(p) \leq w+\epsilon}} \frac{1}{p} \lesssim \frac{\epsilon}{\delta} \sum_{P_{f}(2 \delta)<p \leq P_{f}(\delta)} \frac{1}{p} \approx \frac{\epsilon}{\delta^{2}} \sum_{P_{f}(2 \delta)<p \leq P_{f}(\delta)} \frac{|f(p)|}{p} \ll \frac{\epsilon}{\delta},
$$

by (4.5).

Proof of Lemma 4.1. Fix for the moment $u \in \mathbb{R}$. Given $n \in \mathbb{P}(1, y)$ with $u<f(n) \leq u+\epsilon$, we write $n=a b$, where $a$ is square-free, $b$ is square-full and $(a, b)=1$. We further decompose $a=a_{1} a_{2} a_{3}$, where $a_{1} \in \mathcal{P}\left(1, q_{0}\right), a_{2} \in \mathcal{P}\left(q_{0}, y\right)$ and all primes factors of $a_{3}$ lie in $\mathcal{Q}:=\mathbb{P} \backslash \mathcal{P}$. So

$$
\begin{aligned}
\sum_{\substack{P^{+}(n) \leq y \\
u<f(n) \leq u+\epsilon}} \frac{1}{n}= & \sum_{\substack{P^{+}(b) \leq y \\
b \text { square-full }}} \frac{1}{b} \sum_{\substack{a_{3} \in \mathcal{Q}(1, y) \\
\left(a_{3}, b\right)=1}} \frac{\mu^{2}\left(a_{3}\right)}{a_{3}} \sum_{\substack{a_{2} \in \mathcal{P}\left(q_{0}, y\right) \\
\left(a_{2}, b\right)=1}} \frac{\mu^{2}\left(a_{2}\right)}{a_{2}} \\
& \times \sum_{\substack{u-f\left(a_{2} a_{3} b\right)<f\left(a_{1}\right) \leq u-f\left(a_{2} b\right)+\epsilon \\
a_{1}\left(1, q_{0}\right),\left(a_{1}, b\right)=1}} \frac{\mu^{2}\left(a_{1}\right)}{a_{1}} \\
\leq & \sum_{\substack{P^{+}(b) \leq y \\
b \operatorname{square-full}}} \frac{1}{b} \sum_{a_{3} \in \mathcal{Q}(1, y)} \frac{1}{a_{3}} \sum_{a_{2} \in \mathcal{P}\left(q_{0}, y\right)} \frac{1}{a_{2}} \sup _{v \in \mathbb{R}}\left\{\sum_{\substack{a_{1} \in \mathcal{P}\left(1, q_{0}\right) \\
v<f\left(a_{1}\right) \leq v+\epsilon}} \frac{\mu^{2}\left(a_{1}\right)}{a_{1}}\right\} \\
\ll & \frac{\log y}{\log q_{0}} \cdot \sup _{v \in \mathbb{R}}\left\{\sum_{\substack{a_{1} \in \mathcal{P}\left(1, q_{0}\right) \\
v<f\left(a_{1}\right) \leq v+\epsilon}} \frac{\mu^{2}\left(a_{1}\right)}{a_{1}}\right\} .
\end{aligned}
$$


Next, fix $v \in \mathbb{R}$ and let

$$
\mathcal{N}=\left\{n \in \mathcal{P}\left(1, q_{0}\right): \mu^{2}(n)=1, v<f(n) \leq v+\epsilon\right\} .
$$

As in the proof of Theorem 1.3, we split $\mathcal{N}$ according to the size of $\min \left\{p \mid n: p>q_{J}\right\}$. So we write $\mathcal{N}=\cup_{j=0}^{J} \mathcal{N}_{j}$, where $\mathcal{N}_{0}=\mathcal{N} \cap \mathcal{P}\left(1, q_{J}\right)$ and

$$
\mathcal{N}_{j}=\left\{n \in \mathcal{N}: n=a p b, P^{+}(a) \leq q_{J}<p<P^{-}(b), q_{j}<p \leq q_{j-1}\right\} .
$$

First, we bound $\sum_{n \in \mathcal{N}_{0}} 1 / n$. If $n>1$, then we write $n=m P^{+}(n)=m p^{\prime}$. So we find that

$$
\begin{aligned}
\sum_{n \in \mathcal{N}_{0}} \frac{1}{n} & \leq 1+\sum_{P^{+}(m) \leq q_{J}} \frac{1}{m} \sum_{\substack{p^{\prime} \in \mathcal{P} \cap\left(P^{+}(m), q_{J}\right] \\
v-f(m)<f\left(p^{\prime}\right) \leq v-f(m)+\epsilon}} \frac{1}{p^{\prime}} \\
& \ll 1+\sum_{P^{+}(m) \leq q_{J}} \frac{1}{m}\left(\frac{\epsilon}{2^{J} \delta}+\frac{1}{\log ^{2}\left(1+P^{+}(m)\right)}\right) \ll 1+\frac{\epsilon \log q_{J}}{2^{J} \delta},
\end{aligned}
$$

by applying the second part of (4.3) with $v-f(m), 2^{J} \delta, P^{+}(m)$ and $q_{J}$ in place of $u, \delta, z$ and $w$, respectively.

Next, we bound $\sum_{n \in \mathcal{N}_{j}} 1 / n$ for $j \in\{1, \ldots, J\}$. In this part of the argument we may assume that $J \geq 1$; otherwise, there is no such $j$. Then we have that

$$
\begin{aligned}
\sum_{n \in \mathcal{N}_{j}} \frac{1}{n} & \leq \sum_{b \in \mathcal{P}\left(q_{j}, q_{0}\right)} \frac{1}{b} \sum_{a_{1} \in \mathcal{P}\left(q_{J}^{\lambda}, q_{J}\right)} \frac{1}{a_{1}} \sum_{a_{2} \in \mathcal{P}\left(1, q_{J}^{\lambda}\right)} \frac{\mu^{2}\left(a_{2}\right)}{a_{2}} \sum_{\substack{p \in \mathcal{P} \cap\left(q_{j}, q_{j-1}\right] \\
v<f(p)+f\left(a_{1} b\right)+f\left(a_{2}\right) \leq v+\epsilon}} \frac{1}{p} \\
& \leq \sum_{b \in \mathcal{P}\left(q_{j}, q_{0}\right)} \frac{1}{b} \sum_{a_{1} \in \mathcal{P}\left(q_{J}^{\lambda}, q_{J}\right)} \frac{1}{a_{1}} \sup _{t \in \mathbb{R}}\left\{\sum_{a_{2} \in \mathcal{P}\left(1, q_{J}^{\lambda}\right)} \frac{\mu^{2}\left(a_{2}\right)}{a_{2}} \sum_{\substack{p \in \mathcal{P} \cap\left(q_{j}, q_{j-1}\right] \\
t<f(p)+f\left(a_{2}\right) \leq t+\epsilon}} \frac{1}{p}\right\} .
\end{aligned}
$$

Fix some $t \in \mathbb{R}$ and consider $a_{2} \in \mathcal{P}\left(1, q_{J}^{\lambda}\right)$ and $p \in \mathcal{P} \cap\left(q_{j}, q_{j-1}\right]$ with $t<f\left(a_{2}\right)+f(p) \leq t+\epsilon$, as above. Since $|f(p)| \leq 2^{j} \delta$ for $p \in \mathcal{P} \cap\left(q_{j},+\infty\right)$, by (4.2), we must have that $\left|f\left(a_{2}\right)-t\right| \leq$ $2^{j+1} \delta$. So

$$
\begin{aligned}
\sum_{a_{2} \in \mathcal{P}\left(1, q_{J}^{\lambda}\right)} \frac{\mu^{2}\left(a_{2}\right)}{a_{2}} \sum_{\substack{p \in \mathcal{P} \cap\left(q_{j}, q_{j-1}\right] \\
t<f(p)+f\left(a_{2}\right) \leq t+\epsilon}} \frac{1}{p}=\sum_{\substack{a_{2} \in \mathcal{P}\left(1, q_{J}^{\lambda}\right) \\
\left|f\left(a_{2}\right)-t\right| \leq 2^{j+1} \delta}} \frac{\mu^{2}\left(a_{2}\right)}{a_{2}} \sum_{\substack{p \in \mathcal{P} \cap\left(q_{j}, q_{j-1}\right] \\
t-f\left(a_{2}\right)<f(p) \leq t-f\left(a_{2}\right)+\epsilon}} \frac{1}{p} \\
\ll \frac{\epsilon}{2^{j} \delta} \sum_{\substack{a_{2} \in \mathcal{P}\left(1, q_{J}^{\lambda}\right) \\
\left|f\left(a_{2}\right)-t\right| \leq 2^{j+1} \delta}} \frac{\mu^{2}\left(a_{2}\right)}{a_{2}},
\end{aligned}
$$

by the first part of (4.3) applied with $t-f\left(a_{2}\right), 2^{j-1} \delta, q_{j}$ and $q_{j-1}$ in place of $u, \delta, z$ and $w$, respectively, since $z \geq q_{j} \geq q_{J}=P_{f}\left(2^{J} \delta\right) \geq\left(2^{J} \delta / \epsilon\right)^{\rho} \geq\left(2^{j} \delta / \epsilon\right)^{\rho}$. Finally, if $a_{2}>1$, then we write $a_{2}=m P^{+}\left(a_{2}\right)=m p^{\prime}$. Consequently

$$
\sum_{\substack{a_{2} \in \mathcal{P}\left(1, q_{J}^{\lambda}\right) \\\left|f\left(a_{2}\right)-w\right| \leq 2^{j+1} \delta}} \frac{\mu^{2}\left(a_{2}\right)}{a_{2}} \leq 1+\sum_{m \in \mathcal{P}\left(1, q_{J}^{\lambda}\right)} \frac{1}{m} \sum_{\substack{p^{\prime} \in \mathcal{P} \cap\left(P^{+}(m), q_{J}^{\lambda}\right] \\\left|f\left(p^{\prime}\right)-(t-f(m))\right| \leq 2^{j+1} \delta}} \frac{1}{p^{\prime}}
$$


For every fixed $m \in \mathbb{N}$ we have that

$$
\sum_{\substack{p^{\prime} \in \mathcal{P} \cap\left(P^{+}(m), q_{J}^{\lambda}\right] \\\left|f\left(p^{\prime}\right)-(t-f(m))\right| \leq 2^{j+1} \delta}} \frac{1}{p^{\prime}} \ll \frac{2^{j} \delta}{2^{J} \delta}+\frac{1}{\log ^{2}\left(1+P^{+}(m)\right)},
$$

by (4.3) with $2^{j} \delta, 2^{J} \delta, P^{+}(m)$ and $q_{J}^{\lambda}$ in place of $\epsilon, \delta, z$ and $w$, respectively, and with $u \in\left\{t-f(m)+h \cdot 2^{j} \delta: h \in\{-2,-1,0,1\}\right\}$. So we find that

$$
\begin{aligned}
\sum_{\substack{a_{2} \in \mathcal{P}\left(1, q_{J}^{\lambda}\right) \\
\left|f\left(a_{2}\right)-w\right| \leq 2^{j+1} \delta}} \frac{\mu^{2}\left(a_{2}\right)}{a_{2}} & \ll 1+\sum_{P^{+}(m) \leq q_{J}^{\lambda}} \frac{1}{m}\left(\frac{2^{j} \delta}{2^{J} \delta}+\frac{1}{\log ^{2}\left(1+P^{+}(m)\right)}\right) \\
& \ll 1+\frac{1+\lambda \log q_{J}}{2^{J-j}} \ll 1+\frac{\lambda \log q_{J}}{2^{J-j}} .
\end{aligned}
$$

Combining the above estimate with (4.8) and (4.9) implies that

$$
\begin{aligned}
\sum_{n \in \mathcal{N}_{j}} \frac{1}{n} & \ll \frac{\epsilon}{2^{j} \delta}\left(1+\frac{\lambda \log q_{J}}{2^{J-j}}\right) \sum_{b \in \mathcal{P}\left(q_{j}, q_{0}\right)} \frac{1}{b} \sum_{a_{1} \in \mathcal{P}\left(q_{J}^{\lambda}, q_{J}\right)} \frac{1}{a_{1}} \\
& \ll \frac{\epsilon}{2^{j} \delta}\left(1+\frac{\lambda \log q_{J}}{2^{J-j}}\right) \frac{\log q_{0}}{\log q_{j}} \cdot \frac{1}{\lambda} \leq \frac{\epsilon}{2^{j} \delta}\left(\frac{1}{\lambda}+\frac{\log q_{J}}{2^{J-j}}\right) \frac{\log q_{0}}{\log q_{j}},
\end{aligned}
$$

which, together with relations (4.6) and (4.7), completes the proof of the lemma.

We are now in position to complete the proof of Theorem 1.5 .

Proof of the upper bound in Theorem 1.5. As we have already seen, the function $t \rightarrow g(t)(\log t)^{c}$ is decreasing. In particular, $g$ is strictly decreasing. For every $\delta \in(0,1]$, we define

$$
K^{*}(\delta)=\min \{n \in \mathbb{N}: n \geq 3, g(n) \leq \delta\} .
$$

Then we have that $K^{*}(\delta)-1 \leq K(\delta) \leq 2 K^{*}(\delta)$, with the second inequality being a consequence of Bertrand's postulate.

We claim that

$$
\log \left(K^{*}(\delta)-1\right) \geq \frac{1}{2}\left(\frac{\eta}{\delta}\right)^{1 / c} \log \left(K^{*}(\eta)-1\right) \quad(0<\eta \leq \delta \leq 1) .
$$

Indeed, if $K^{*}(\delta)=K^{*}(\eta)$, then this inequality holds trivially. Next, assume that $K^{*}(\eta) \geq$ $K^{*}(\delta)+1 \geq 4$. Then the definition of $K^{*}(\eta)$ implies that $g\left(K^{*}(\eta)-1\right)>\eta$. Since, in addition, the function $t \rightarrow g(t)(\log t)^{c}$ is decreasing and $(x-1) \leq x^{2}$ for all $x \geq 3$, we find that

$$
\begin{aligned}
1 \geq \frac{g\left(K^{*}(\eta)-1\right)\left(\log \left(K^{*}(\eta)-1\right)\right)^{c}}{g\left(K^{*}(\delta)\right)\left(\log K^{*}(\delta)\right)^{c}} & \geq \frac{\eta\left(\log \left(K^{*}(\eta)-1\right)\right)^{c}}{\delta\left(\log K^{*}(\delta)\right)^{c}} \\
& \geq \frac{\eta\left(\log \left(K^{*}(\eta)-1\right)\right)^{c}}{\delta\left(2 \log \left(K^{*}(\delta)-1\right)\right)^{c}} .
\end{aligned}
$$

In any case, (4.10) holds.

Using relation (4.10), we shall show that we may apply Lemma 4.1 with $P_{f}=K^{*}-1$, $\mathcal{P}, \lambda=1 / A$ and $\rho=2$. Condition (4.1) holds by assumption and condition (4.2) follows immediately by the definition of $K^{*}$ and the fact that $|f(p)| \leq g\left(K^{*}(\delta)\right) \leq \delta$ for $p \geq K^{*}(\delta)$. Lastly, we show (4.3) with $\lambda=1 / A$ and $\rho=2$. This will be done in several steps. Fix $u \in \mathbb{R}$, $0<\eta \leq \delta \leq 1$ and $2 \leq z \leq w \leq \min \left\{P_{f}(\delta), P_{f}(\eta)^{1 / A}\right\}$. 
First, we show (4.3) when $z \geq w^{1 / 4}$. By assumption, there is an absolute constant $C>0$ such that

$$
\left|f\left(p_{1}\right)-f\left(p_{2}\right)\right| \geq \frac{2}{C} \min \left\{\frac{g\left(p_{2}\right)\left(p_{2}-p_{1}\right)}{p_{2} \log p_{2}}, g\left(p_{2}^{A}\right)\right\} \quad\left(p_{1}<p_{2}, p_{1}, p_{2} \in \mathbb{P}\right) .
$$

We claim that if $v \in \mathbb{R}$ and $\eta^{\prime}:=\min \{\eta, \delta / \log w\} / C$, then

$$
\sum_{\substack{p \in \mathcal{P} \cap(z, w] \\ v<f(p) \leq v+\eta^{\prime}}} \frac{1}{p} \ll \begin{cases}\eta^{\prime} / \delta & \text { if } z \geq P_{f}(2 \delta) \geq(2 \delta / \eta)^{2}, \\ \eta^{\prime} / \delta+1 /(\log w)^{3} & \text { otherwise. }\end{cases}
$$

If this relation does hold, then breaking the interval $(u, u+\eta]$ into at most $1+\eta / \eta^{\prime} \leq$ $1+C \log w$ intervals of the form $\left(v, v+\eta^{\prime}\right.$, we deduce that (4.3) holds too when $z \geq w^{1 / 4}$. So it remains to show (4.11) to complete the proof of (4.3) in this special case.

Without loss of generality, we may assume that $w \geq 3$; otherwise there are no primes in $(z, w] \subset(2,3)$ and (4.11) is trivially true. In particular, we may assume that $K^{*}(\eta) \geq$ $K^{*}(\delta) \geq 4$. Therefore, for every $p \in(z, w]$, we have that $g(p) \geq g\left(K^{*}(\eta)-1\right)>\eta$ and $g\left(p^{A}\right) \geq g\left(K^{*}(\delta)-1\right)>\delta$. Now, consider two primes $p_{1}<p_{2}$ that both belong to the set $\left\{p \in \mathcal{P} \cap(z, w]: v<f(p) \leq v+\eta^{\prime}\right\}$. Then

$$
\begin{aligned}
\frac{1}{C} \min \left\{\eta, \frac{\delta}{\log w}\right\}=\eta^{\prime} & >\left|f\left(p_{1}\right)-f\left(p_{2}\right)\right| \\
& \geq \frac{2}{C} \min \left\{\frac{g\left(p_{2}\right)\left(p_{2}-p_{1}\right)}{p_{2} \log p_{2}}, g\left(p_{2}^{A}\right)\right\} \\
& \geq \frac{2}{C} \min \left\{\frac{\delta\left(p_{2}-p_{1}\right)}{p_{2} \log p_{2}}, \eta\right\}
\end{aligned}
$$

and, consequently,

$$
0<p_{2}-p_{1} \leq \frac{C \eta^{\prime}}{2 \delta} \cdot p_{2} \log p_{2}=\min \left\{\frac{\eta}{\delta}, \frac{1}{\log w}\right\} \cdot \frac{p_{2} \log p_{2}}{2} \leq \frac{p_{2}}{2} .
$$

Set

$$
P=\max \left\{p \in \mathcal{P} \cap(z, w]: v<f(p) \leq v+\eta^{\prime}\right\}
$$

and

$$
y=\frac{C \eta^{\prime}}{2 \delta} \cdot P \log P \leq \frac{P}{2}
$$

so that $\left\{p \in \mathcal{P} \cap(z, w]: v<f(p) \leq v+\eta^{\prime}\right\} \subset[P-y, P]$. The second part of relation (4.11) then follows by the Prime Number Theorem [13, Theorem 1, p. 167]. For the first part of (4.11), note that if $z \geq P_{f}(2 \delta) \geq(2 \delta / \eta)^{2}$, then

$$
\frac{y}{\sqrt{P}}=\frac{\sqrt{P} \log P}{2} \min \left\{\frac{\eta}{\delta}, \frac{1}{\log w}\right\} \geq \frac{(2 \delta / \eta) \log z}{2} \min \left\{\frac{\eta}{\delta}, \frac{1}{\log w}\right\} \geq \frac{1}{4},
$$

where we used our assumption that $z \geq \max \left\{w^{1 / 4}, 2\right\}$. So the first part of (4.11) follows by the Brun-Titchmarsch inequality [13, Theorem 9, p. 73], thus completing the proof of (4.11) and hence of (4.3) in the case when $z \geq w^{1 / 4}$.

Finally, we show (4.3) when $z<w^{1 / 4}$. First, note that

$$
\log P_{f}\left(2^{j} \delta\right) \geq 2^{-1-j / c} \log P_{f}(\delta) \geq 2^{-1-j / c} \log w \geq 4^{-j} \log w,
$$


by (4.10), for every $j \geq 1$. Since $w \leq P_{f}(\delta)$ too, by assumption, we deduce that

$$
P_{f}\left(2^{j} \delta\right) \geq w_{j}:=w^{4^{-j}} \quad(j \geq 0) .
$$

Applying this inequality with $j=1$ implies that $z<P_{f}(2 \delta)$, that is to say we are in the second case of (4.3). Let

$$
\begin{gathered}
j_{0}=\max \left\{j \geq 0: w_{j} \geq z \text { and } 2^{j} \leq 1 / \delta\right\}, \\
S_{j}=\sum_{\substack{p \in \mathcal{P} \cap\left(w_{j+1}, w_{j}\right] \\
u<f(p) \leq u+\eta}} \frac{1}{p},
\end{gathered}
$$

for $j \in\left\{0,1, \ldots, j_{0}-1\right\}$, and

$$
S_{j_{0}}=\sum_{\substack{p \in \mathcal{P} \cap\left(z, w_{j_{0}}\right] \\ u<f(p) \leq u+\eta}} \frac{1}{p}
$$

Then the part of (4.3) that we have already proven and (4.13) imply that

$$
S_{j} \ll \frac{\eta}{2^{j} \delta}+\frac{16^{j}}{(\log w)^{2}},
$$

for $j \in\left\{0,1, \ldots, j_{0}-1\right\}$. We claim that the same estimate holds for $S_{j_{0}}$ too. If $2^{j_{0}+1} \delta \leq 1$, then $w_{j_{0}}^{1 / 4}=w_{j_{0}+1}<z$ and thus we may apply again the part of (4.3) that we have already proven. Finally, if $2^{j_{0}+1} \delta>1$, then we have that $w_{j_{0}} \leq P_{f}\left(2^{j_{0}} \delta\right) \leq P_{f}(1 / 2) \ll 1$, since $g(t) \ll 1 /(\log t)^{c}$ by our assumptions on $f$. Consequently, covering the interval $\left(z, w_{j_{0}}\right]$ by $O(1)$ intervals of the form $\left(t, t^{4}\right]$ and applying the already proven part of (4.3) shows that (4.14) holds in this case too for $j=j_{0}$. Summing (4.14) over $j \in\left\{0,1, \ldots, j_{0}\right\}$ implies that

$$
\sum_{\substack{p \in \mathcal{P} \cap(z, w] \\ u<f(p) \leq u+\eta}} \frac{1}{p}=\sum_{j=0}^{j_{0}} S_{j} \ll \frac{\eta}{\delta}+\frac{1}{(\log z)^{2}},
$$

which completes the proof of (4.3). In conclusion, we may apply Theorem 4.1 with $P_{f}=$ $K^{*}-1, \rho=2$ and $\lambda=1 / A$.

We are finally ready to show the upper bound in Theorem 1.5. Let $\epsilon \in(0,1 / 2]$. We may assume that $K^{*}(\epsilon)$ is large enough; otherwise, the theorem follows by the trivial upper bound $Q_{\mathcal{F}_{y}}(\epsilon) \leq 1$. In particular, we may assume that the parameter $\delta:=g\left(\left\lfloor K^{*}(\epsilon)^{1 / A}\right\rfloor-1\right)$ lies in $[\epsilon, 1 / 2]$. Since $g$ is strictly decreasing, the definition of $K^{*}$ implies that

$$
K^{*}(\delta)=\left\lfloor K^{*}(\epsilon)^{1 / A}\right\rfloor-1 .
$$

In particular, $P_{f}(\delta) \leq P_{f}(\epsilon)^{1 / A}$. For $j \in \mathbb{N} \cup\{0\}$ with $2^{j} \leq 1 / \delta$, we set $q_{j}=P_{f}\left(2^{j} \delta\right)=$ $K^{*}\left(2^{j} \delta\right)-1$. Note that

$$
q_{0}=K^{*}(\delta)-1 \leq K^{*}(\epsilon)-1 \leq K(\epsilon)
$$

and

$$
\log q_{j} \geq 2^{-1-(j-i) / c} \log q_{i} \quad(0 \leq i \leq j \leq \log (1 / \delta) / \log 2)
$$

by (4.10). Set

$$
J=\max \left(\{0\} \cup\left\{j \in \mathbb{N}: 2^{j} \leq 1 / \delta \text { and } q_{j} \geq\left(2^{j} \delta / \epsilon\right)^{2}\right\}\right) .
$$


Then Theorem 4.1 and relation (4.16) imply that, for $y \geq K(\epsilon) \geq q_{0}$, we have that

$$
\begin{aligned}
Q_{\mathcal{F}_{y}}(\epsilon) & \ll A \frac{1}{\log q_{0}}+\frac{\epsilon}{\delta} \sum_{j=1}^{J} \frac{1}{2^{j} \log q_{j}}+\frac{\epsilon}{\delta} \sum_{j=0}^{J} \frac{\log q_{J}}{2^{J} \log q_{j}} \\
& \ll \frac{1}{\log q_{0}}+\frac{\epsilon}{\delta} \sum_{j=1}^{J} \frac{1}{2^{j-j / c} \log q_{0}}+\frac{\epsilon}{\delta} \sum_{j=0}^{J} \frac{\log q_{J}}{2^{J-j / c} \log q_{0}} \\
& \ll \frac{\min \{1 /(c-1), 1+J \epsilon / \delta\}}{\log q_{0}}+\frac{\epsilon}{\delta} \frac{\log q_{J}}{2^{J(1-1 / c)} \log q_{0}} \\
& \ll \frac{\min \{1 /(c-1), 1+J \epsilon / \delta\}}{\log q_{0}}+\frac{\epsilon}{\delta} \frac{\log q_{J}}{\max \{1,(J+1)(c-1)\} \log q_{0}} .
\end{aligned}
$$

Finally, note that if $2^{J+1} \leq 1 / \delta$, then the maximality of $J$ and (4.16) imply that

$$
\log q_{J} \leq 4 \log q_{J+1} \leq 8 \log \left(2^{J+1} \delta / \epsilon\right) \ll J+1+\log (\delta / \epsilon) .
$$

On the other hand, if $2^{J+1}>1 / \delta$, then $q_{J} \leq K^{*}(1 / 2) \ll 1$, since $g(t) \ll(\log t)^{-c}$. In any case, we find that $\log q_{J} \ll J+1+\log (\delta / \epsilon)$. So the inequalities

$$
\frac{J \epsilon}{\delta} \ll \frac{\epsilon \log (1 / \delta)}{\delta} \leq \log (1 / \epsilon) \text { and } \quad \frac{\epsilon}{\delta} \log (\delta / \epsilon) \ll 1
$$

and relation (4.17) imply that

$$
Q_{\mathcal{F}_{y}}(\epsilon) \ll \frac{\min \{1 /(c-1), \log (1 / \epsilon)\}}{\log q_{0}} .
$$

Finally, we have that $\log q_{0} \asymp_{A} \log K^{*}(\epsilon) \asymp \log K(\epsilon)$, by (4.15) and the fact that $K^{*}-1 \leq$ $K \leq 2 K^{*}$. So the upper bound in Theorem 1.5 follows.

\section{ACKNOWLEDGENTS}

I would like to thank Gérald Tenenbaum for pointing out relation (1.4) to me. I am also grateful to Régis de la Bretèche and Maksym Radziwill for some helpful comments. In addition, I would like to thank the referees who handled the paper, as their comments exposed some inaccuracies and improved the exposition of the main ideas. This paper was largely written while visiting Université Paris-Diderot, which I would like to thank for its hospitality. During that time I was a postdoctoral fellowship at the Centre de recherches mathématiques at Montréal, which I would like to thank for the financial support.

\section{REFERENCES}

1. R. de la Bretèche and G. Tenenbaum, Sur la concentration de certaines fonctions additives, Math. Proc. Cambridge Philos. Soc. 152 (2012), no. 1, 179-189. Sur la concentration de certaines fonctions additives - corrigendum. Math. Proc. Cambridge Philos. Soc. 152 (2012), no. 1, 191.

2. H. Diamond and D. Rhoads, The modulus of continuity of the distribution function of $\phi(n) / n$. Topics in classical number theory, Vol. I, II (Budapest, 1981), 335-353, Colloq. Math. Soc. János Bolyai, 34, North-Holland, Amsterdam, 1984.

3. P. D. T. A. Elliott, Probabilistic number theory. I. Mean-value theorems. Grundlehren der Mathematischen Wissenschaften [Fundamental Principles of Mathematical Science], 239, Springer-Verlag, New York-Berlin, 1979. 
4. P. D. T. A. Elliott, Probabilistic number theory. II. Central limit theorems. Grundlehren der Mathematischen Wissenschaften [Fundamental Principles of Mathematical Sciences], 240, Springer-Verlag, Berlin-New York, 1980.

5. P. Erdős, On the distribution function of additive functions. Ann. of Math. (2) 47, (1946). 1-20.

6. P. Erdös, On the distribution of numbers of the form $\sigma(n) / n$ and on some related questions. Pacific J. Math. 52 (1974), 59-65.

7. P. Erdős and I. Kátai, On the concentration of distribution of additive functions. Acta Sci. Math. (Szeged) 41 (1979), no. 3-4, 295-305.

8. P. Erdős and A. Wintner, Additive arithmetical functions and statistical independence. Amer. J. Math. 61 (1939), 713-721.

9. G. Halász, On the distribution of additive arithmetic functions. Collection of articles in memory of Jurii Vladimirovic Linnik. Acta Arith. 27 (1975), 143-152.

10. R. R. Hall and G. Tenenbaum, Divisors. Cambridge Tracts in Mathematics, vol. 90, Cambridge University Press, Cambridge, 1988.

11. J. Kubilius, Probabilistic methods in the theory of numbers. Translations of Mathematical Monographs, Vol. 11 American Mathematical Society, Providence, R.I. 1964.

12. I. Z. Ruzsa, On the concentration of additive functions. Acta Math. Acad. Sci. Hungar. 36 (1980), no. 3-4, 215-232 (1981).

13. G. Tenenbaum, Introduction to analytic and probabilistic number theory. Translated from the second French edition (1995) by C. B. Thomas. Cambridge Studies in Advanced Mathematics, 46. Cambridge University Press, Cambridge, 1995.

14. M. M. Tjan, On the question of the distribution of values of the Euler function $\phi(n)$. (Russian. Lithuanian, French summary) Litovsk. Mat. Sb. 6 (1966), 105-119.

Département de mathématiques et de statistique, Université de Montréal, CP 6128 succ. Centre-Ville, Montréal, Québec H3C 3J7, Canada

E-mail address: koukoulo@dms.umontreal.ca 